\title{
Evaluation of Whole-Body Vibration and Ride Comfort in a Passenger Car
}

\author{
Hassan Nahvi \\ Mechanical Eng. Dept., Isfahan University of Tech., Isfahan 84156-83111, Iran \\ Mohammad Hosseini Fouladi and Mohd Jailani Mohd Nor \\ MEMS-Automotive Research Group, Dept. of Mechanical and Materials Eng., Faculty of Engineering and Built \\ Environment, Universiti Kebangsaan Malaysia, 43600 Bangi, Selangor, Malaysia
}

(Received 23 August 2008; revised 26 May 2009; accepted 11 June 2009)

\begin{abstract}
Whole-body vibration transmission influences comfort, performance, and long-term health of the driver. This current study is an objective evaluation of vehicle comfort characteristics based on standard mathematical formulae and frequency analyses. A variety of road types were selected and quantified by using the International Roughness Index (IRI). To assess vibrations transmitted to the passengers, vibration dose values (VDV), kurtosis, frequency response functions (FRF), and power spectral densities (PSD) of the compartment recorded signals were evaluated. SEAT values based on VDV outputs qualified the seat suspension as a vibration isolator, whereas the FRF and PSD quantified that behaviour through frequency analyses. Results indicate that energy concentration is at frequencies lower than $30 \mathrm{~Hz}$. Such low frequency excitations are well attenuated by seat suspension in the vertical direction but are amplified (up to five times in harsh conditions) by a backrest in the fore-aft trend. Signals are amplified beyond $30 \mathrm{~Hz}$, but amplitudes are still very low. It seems that backrest assembly still can be improved to become a better isolator. However, $T_{15}$ (time to reach severe discomfort), even in harsh conditions, is more than three hours, which exhibits the overall good quality of the vehicle suspension systems. Kurtosis and VDV correlate with IRI and may be used as two objective metrics, together with jury evaluation, to create a vehicle vibration-comfort index in the future.
\end{abstract}

\section{INTRODUCTION}

Vibration transmission to passengers has a large influence on comfort, performance, and health. ${ }^{1}$ A comfortable ride is essential for a vehicle to obtain passenger satisfaction. Because of this, vehicle manufacturers are constantly seeking to improve vibration comfort. Many factors influence the transmission of vibration to and through the body. Transmission associated with the dynamic system depends on the frequency and direction of the input motion and the characteristics of the seat from which the vibration exposure is received. Vibrations up to $12 \mathrm{~Hz}$ affect all of the human organs, while those above $12 \mathrm{~Hz}$ have local effects. ${ }^{2}$ Low-frequency $(4-6 \mathrm{~Hz})$ cyclic motions, like those caused by tires rolling over an uneven road, can resonate the body. Just one hour of seated vibration exposure may cause muscle fatigue and make the user more susceptible to back injury.

This paper is a subdivision of a general research conducted to evaluate vibro-acoustical comfort inside the vehicle compartment. The first part was to define a vehicle acoustical comfort index using objective and subjective evaluations. ${ }^{3}$ This paper is an objective evaluation of vehicle vibration comfort, which is the first step of the vibration comfort assessment. Analysis of road conditions parameters, such as the International Roughness Index (IRI), and their correlation with kurtosis and the vibration dose value (VDV) can give useful information about the effect of road roughness on passenger vibration comfort.

Further research may include a subjective vibration evaluation (jury test), and the results of the subjective and objective as- sessments may be used to define an index for vehicle vibration comfort. This index eliminates the need for further subjective estimations and can be a useful parameter in various correlation analyses and vibration comfort predictions. It is believed that specific results of a vibration comfort index are only valid for the exact vehicle type. Still, different manufacturers can use the same method to derive the vibration comfort index for their products. Methods and general results of the current research (like correlations conducted for VDV, IRI, kurtosis, and velocity) are applicable for other researches as well.

Human responses to whole-body vibration can be evaluated by two main standards - the British Standard 6841 (BS 6841) (1987) and the International Standard 2631 (ISO 2631) (1997). The BS 6841 considers a frequency range of $0.5-80 \mathrm{~Hz} .^{4}$ As shown in Fig. 1, this standard recommends measuring four axes of vibration on the seat (fore-aft, lateral, and vertical vibration on the seat surface as well as fore-aft vibration at the backrest) and combines them in an evaluation that assesses vibration severity. The ISO 2631 suggests vibration measurements in the three translational axes on the seat pan, but only the axis with the greatest vibration is used to estimate vibration severity. $^{5}$

The current trend in vibration research is to use multi-axis values. This may be seen in studies (such as those by Paddan and Griffin $^{6}$ and Hinz et al. ${ }^{7}$ ). Huston and Zhao examined how the shape, frequency, and amplitude of mechanical shocks affect the comfort response of the seated human. ${ }^{8}$ Recently, the effects caused by different experimental design variables on subjective response and vibration accelerations were investigated by Jonsson and Johansson. ${ }^{9}$ In this study, ride comfort and vi- 